\title{
A Floating Differential DAC Expands the Output Voltage Range for Electrochemical Measurements
}

\author{
Norbert Sailer* ${ }^{* \dagger}$, Inge Siegl ${ }^{* \dagger}$, Markus Haberler* ${ }^{* \dagger}$, Christoph Steffan ${ }^{\dagger}$ \\ Email: norbert.sailer@student.tugraz.at, inge.siegl@student.tugraz.at \\ *Institute of Electronics, Graz University of Technology \\ ${ }^{\dagger}$ Infineon Technologies Austria AG, Development Center Graz
}

\begin{abstract}
To operate a variety of electrochemical sensors, a potentiostat requires a large output voltage range. In order to implement this with a fully integrated potentiostat, we expand the digital-to-analog converter's (DAC) output voltage range by providing a floating reference voltage with a current mirror and two amplifiers in a negative feedback configuration. A segmented 16 bit resistor string (R-string) DAC uses the floating reference voltage to generate excitation signals within the supply voltage range. We show that our floating differential DAC supports an output voltage range from $0.32 \mathrm{~V}$ to $2.61 \mathrm{~V}$ when operated at a supply voltage of $3 \mathrm{~V}$. Furthermore, the static and the dynamic performance of the DAC are maintained during output voltage range expansion. These properties suggest that our DAC is suitable for integration in an electrochemical measurement system.
\end{abstract}

Index Terms-Digital-to-analog converter (DAC), electrochemical measurement, excitation signal, output voltage range, pointof-care (POC), segmented resistor string

\section{INTRODUCTION}

Fast, portable, and low-cost measurement solutions are needed for point-of-care (POC) diagnostics. These diagnostic methods can be based on electrochemical measurements. Electrochemical measurement techniques are performed by an electronic device called potentiostat. The potentiostat applies a differential excitation voltage $V_{\text {cell }}$ between two electrodes of an electrochemical sensor, the working, and the reference electrode. In the three-electrode configuration, the control loop of the reference electrode is closed over the sensor and sample using a third electrode, the counter electrode [1]. The required voltages between the electrodes therefore depend on the electrochemical sensor and determine the required output voltage range of the potentiostat's digital-to-analog converter (DAC).

By supporting many electrochemical sensor types, such as the microneedle-based biosensor developed in the H2020 ELSAH project [2], integrated CMOS potentiostats with a wide output voltage range foster POC diagnostics development. This can reduce production and development costs. However, up to now, potentiostats are often designed for a specific electrochemical sensor and hence, support only a limited output voltage range.

This research is part of the ELSAH-project, which has received funding from the European Union's Horizon 2020 research and innovation program under grant agreement No 825549 .

\section{A. State-of-the-Art Output Voltage Range Restrictions}

The majority of integrated potentiostats generate the differential excitation voltage $V_{\text {cell }}$ with a single DAC, while the second electrode is connected to a fixed potential. In [3]-[6], the potential of the working electrode is fixed in the middle of the supply voltage range, thus limiting the output voltage range to less than half of the supply voltage. References [7]-[9] use one of the supply rails as a fixed potential. This limits the cell voltage to one polarity. Often, the value of the second electrode's potential is not explicitly stated [8]-[22]. Even potentiostat architectures with two DACs [5], [23]-[25] seem to keep one electrode during the measurement at a fixed potential.

In summary, setting one of the potentiostat electrodes to a fixed potential restricts the output voltage range of the potentiostat, either in range or in polarity. Therefore, the number of supported electrochemical sensors and applications, that could theoretically be possible with a given supply voltage, is limited.

\section{B. Efficient Output Voltage Range Exploitation}

A DAC architecture for potentiostats comprised of two DACs presented in [26] exploits the output voltage range more efficiently than conventional architectures. The two DACs set not only the differential voltage $V_{\text {cell }}$, but also control the absolute electrode potential relative to the system's ground potential during electrochemical measurements. This dynamic electrode positioning at runtime exploits the output voltage range more efficiently because all three electrodes can float within the supply voltage range.

However, signal generation with two independent DACs is complicated. Digital control of $V_{\text {cell }}$ is inconvenient since the differential voltage and its absolute potential cannot be adjusted independently. Furthermore, both DACs require a high resolution in order to avoid significant inaccuracies. This results in hardware overhead.

We therefore propose a floating differential DAC for integrated potentiostats with dynamic electrode positioning. The DAC supports a high output voltage range, almost reaching the supply rails, and versatile excitation signal capabilities.

\section{DAC DESIGN CONSIDERATIONS}

In order to implement dynamic electrode positioning, as demonstrated in [26], the DAC has to be able to generate 


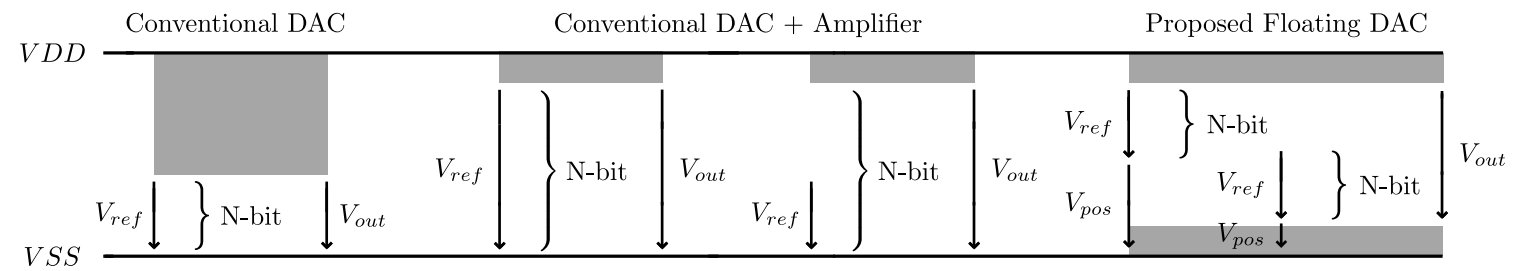

Fig. 1. DAC output voltage range comparison. The output voltage range $V_{\text {out }}$ of a conventional DAC is limited by the reference voltage $V_{r e f}$. Amplifying $V_{\text {ref }}$ or the DAC output voltage expands $V_{\text {out }}$, but decreases the resolution. Our proposed DAC can float within the supply voltage range by adding an adjustable positioning voltage $V_{\text {pos }}$. The DACs resolution is available over the whole output voltage range.

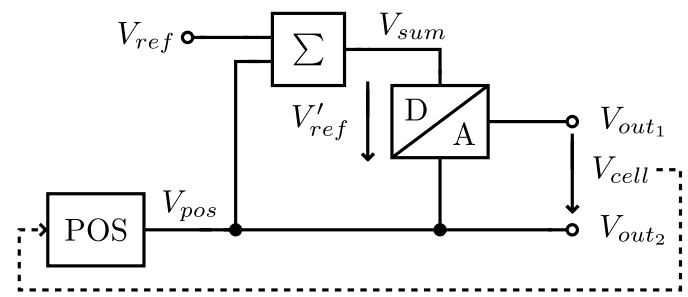

Fig. 2. Block diagram of the proposed floating differential DAC. The positioning voltage $V_{\text {pos }}$ is set by feedback (dashed line). Adding $V_{\text {pos }}$ and the reference voltage $V_{\text {ref }}$ generates a floating reference voltage for the DAC.

output voltages over the whole supply voltage range.

\section{A. DAC Output Voltage Range}

The output voltage range $V_{\text {out }}$ of conventional DACs is limited by the amplitude of the reference voltage $V_{\text {ref }}$. Amplifying either $V_{\text {ref }}$ or the generated output voltage expands the output voltage range but decreases the voltage resolution, as shown in Fig. 1. The resolution can be increased at the expense of hardware overhead and design complexity.

The proposed design generates a floating reference voltage $V_{\text {ref }}$ to expand the output range. A voltage adder circuit shifts $V_{\text {ref }}$ up by the adjustable positioning voltage $V_{\text {pos }}$. Fig. 1 illustrates that the DACs resolution is available over the whole output voltage range.

The block diagram of this architecture is depicted in Fig. 2. A feedback loop that controls $V_{\text {pos }}$ realizes dynamic electrode positioning to adjust $V_{\text {cell }}$ adequately within the supply voltage range. Adding the voltages $V_{\text {pos }}$ and $V_{\text {ref }}$ generates a new floating reference voltage $V_{r e f}^{\prime}$ for the DAC.

\section{B. DAC Output Signal Capabilities}

Electrochemical measurement techniques differ in their excitation voltage waveform. Constant voltages, step functions as well as sinusoidal excitation signals are used.

In order to support as many electrochemical measurement techniques as possible our proposed DAC can generate all three mentioned excitation signals over a wide output voltage range.

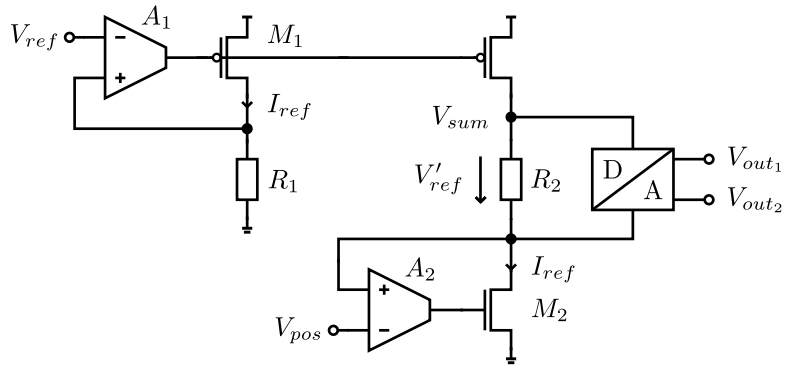

Fig. 3. Simplified schematic of the voltage adder block. The reference voltage $V_{\text {ref }}$ is converted into a current $I_{\text {ref }}$ and mirrored into a second branch Amplifier $A_{2}$ and transistor $M_{2}$ add the positioning voltage $V_{\text {pos }}$.

\section{FloAting DifFERENTIAL DAC}

A current mirror based adder generates the floating reference voltage for the DAC. The DAC is based on a segmented resistor string (R-string) architecture. When biased with a constant current, the voltage drops across the R-string are independent of the absolute R-string potentials. Thus, this architecture can float within the supply voltage range. In addition, R-string DACs are suitable for low-power designs as required by POC diagnostic devices.

Compared to a conventional DAC, the segmented architecture reduces the number of resistors and switches. In addition, signal generation complexity can be reduced if the first stage generates the dc component and the second stage the ac component of the excitation signal [27].

\section{A. Voltage Adder}

The simplified schematic of the voltage adder is depicted in Fig. 3. Amplifier $A_{1}$, transistor $M_{1}$ and resistor $R_{1}$ convert the reference voltage $V_{\text {ref }}$ into a current $I_{\text {ref }}$. A matched transistor pair mirrors $I_{\text {ref }}$ into a second branch. Amplifier $A_{2}$ and transistor $M_{2}$ regulate the bottom potential of resistor $R_{2}$ to $V_{\text {pos }}$. The voltage drop across $R_{2}$ adds $V_{\text {ref }}$ if $R_{1}$ and $R_{2}$ are matched. The potential of node $V_{\text {sum }}$ becomes

$$
V_{\text {sum }}=V_{\text {ref }}+V_{\text {pos }} .
$$




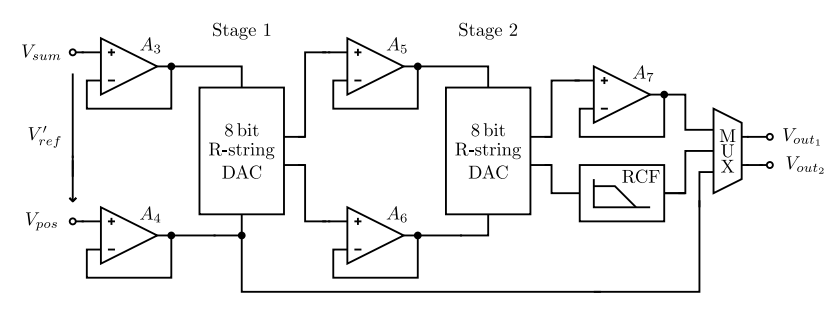

Fig. 4. Simplified schematic of the segmented 16 bit floating differential DAC. Two identical 8 bit resistor string (R-string) DACs are decoupled by operational amplifiers $\left(A_{3}\right.$ to $\left.A_{6}\right)$. The generated voltage can be buffered by amplifier $A_{7}$ or low-pass filtered by the reconstruction filter (RCF). An analog multiplexer (MUX) selects the output signals.

\section{B. Segmented R-string DAC}

The simplified schematic of the segmented 16 bit R-string DAC is depicted in Fig. 4. Amplifiers $A_{3}$ to $A_{6}$ decouple the two identical 8 bit R-string DACs. The individual DACs are comprised of 256 polysilicon resistors and two 8 bit binary decoders. Amplifier $A_{7}$ buffers dc and step signals, whereas the reconstruction filter (RCF) low-pass filters and buffers sinusoidal signals. The reconstruction filter is implemented by an active $2^{\text {nd }}$-order low-pass unity-gain Sallen-Key filter and is designed for a signal bandwidth of $10 \mathrm{kHz}$. An analog multiplexer (MUX) selects the output signals.

\section{Realization}

Our proposed floating differential DAC is part of an integrated potentiostat [28] and manufactured in a $130 \mathrm{~nm}$ standard CMOS process. The DAC is designed for a supply voltage of $3 \mathrm{~V}$, but can operate at supply voltages down to $2 \mathrm{~V}$. Decreasing the supply voltage decreases the DACs output voltage range. The DACs reference voltage is $1.2 \mathrm{~V}$.

A micrograph of the manufactured integrated potentiostat with a layout overlay is shown in Fig. 5. The layout of the DAC and the positioning circuit are shown in more detail. In total, the two circuits cover an area of approximately $0.14 \mathrm{~mm}^{2}$.

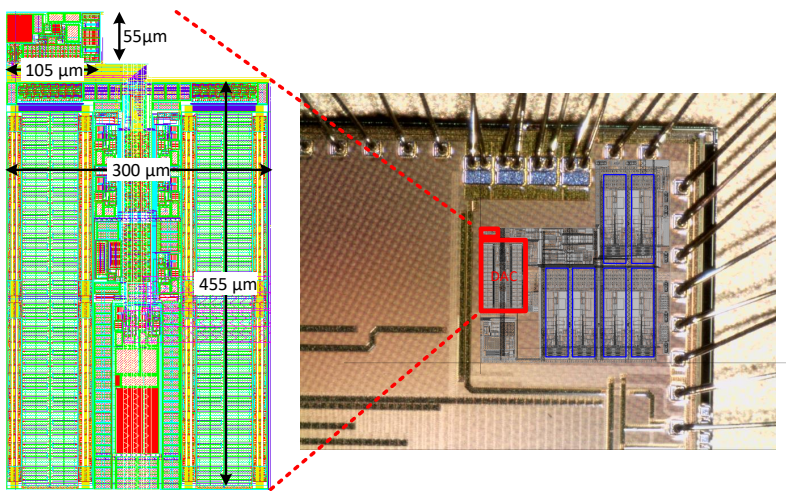

Fig. 5. Micrograph of the manufactured integrated potentiostat [28] with a layout overlay. The DAC and the positioning circuit are shown in more detail. They cover an area of approximately $0.14 \mathrm{~mm}^{2}$.

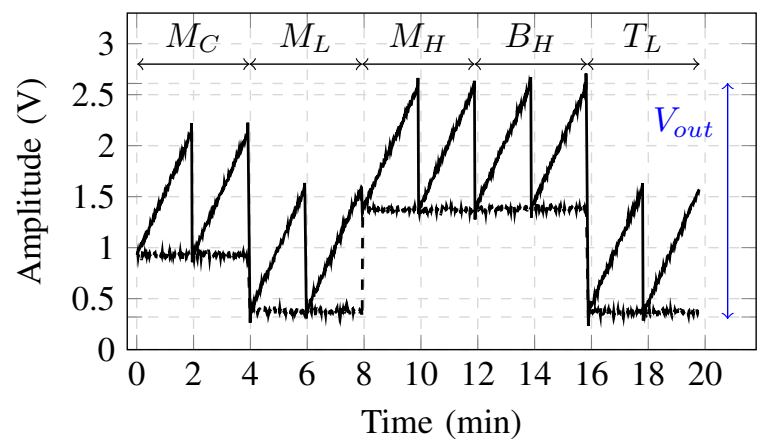

Fig. 6. Measured test vector for DNL and INL characterization. The plot shows the absolute electrode potentials of the working and reference electrode which define $V_{\text {cell }}$. Our floating DAC supports an output voltage range $V_{\text {out }}$ from $0.32 \mathrm{~V}$ to $2.61 \mathrm{~V}$ when operated with a supply voltage of $3 \mathrm{~V}$.

\section{Measurement Results}

We measured 10 chips to characterize the static and dynamic performance of our proposed floating differential DAC.

The transfer functions for both 8 bit R-string DACs were measured independently after each other. By varying the positioning voltage $V_{\text {pos }}$, the DAC was shifted within the output voltage range. The measured output voltages are shown in Fig. 6. As illustrated in Fig. 6, our floating DAC supports an output voltage range $V_{\text {out }}$ from $0.32 \mathrm{~V}$ to $2.61 \mathrm{~V}$ when operated with a supply voltage of $3 \mathrm{~V}$.

\section{A. Static Performance}

The differential nonlinearity (DNL) and integral nonlinearity (INL) performances of each R-string DAC were calculated from the measured transfer functions. Fig. 7 shows the DNL and INL as a function of the digital input code of one chip for three different values of $V_{\text {pos }}$ and three different feedback positions of amplifier $A_{2}$ (top, middle, and bottom of $R_{2}$ ). The measurements confirm that the dynamic positioning within the supply voltage range does not influence the static performance. Asymmetry in the layout causes the deviation between the two R-strings.

Histograms with accumulated results of 10 chips are shown in Fig. 8. Over all operating conditions the DAC achieves a DNL within -0.26 LSB and 0.15 LSB, and an INL within $-0.26 \mathrm{LSB}$ and $0.11 \mathrm{LSB}$. Again, the measurements confirm that our proposed floating architecture does not introduce significant nonlinearities.

\section{B. Dynamic Performance}

The signal-to-noise-and-distortion ratio (SNDR) as a function of the DAC's output frequency is plotted in Fig 9. Measurements were done for two chips, one with and the other without dynamic electrode positioning. The SNDR is nearly identical over the observed frequency range in both cases. Hence, output voltage range expansion does not influence the DAC's dynamic performance.

Sinusoidal signals with amplitudes of $-6 \mathrm{dBFS}$ and frequencies from $3 \mathrm{~Hz}$ to $10 \mathrm{kHz}$ were generated for the dynamic performance characterization of the DAC. Stage 1 set the dc 


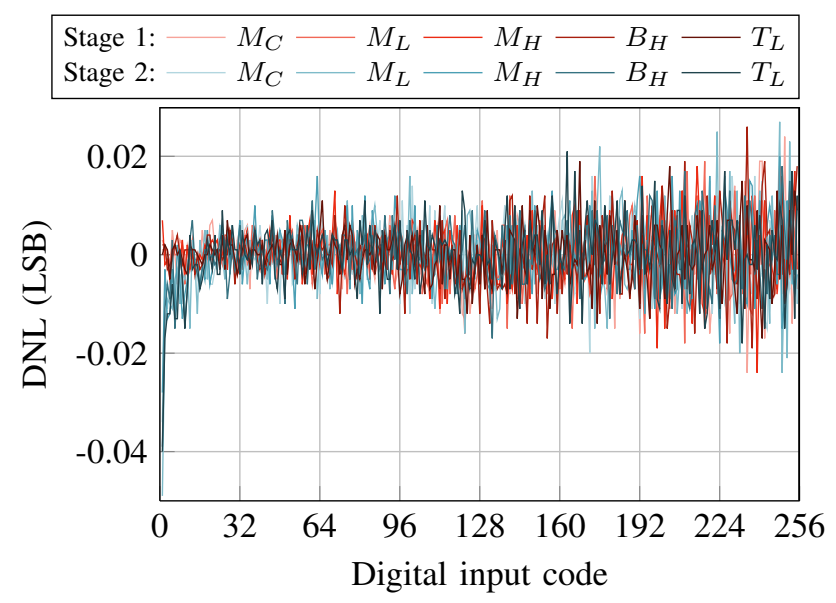

(a)

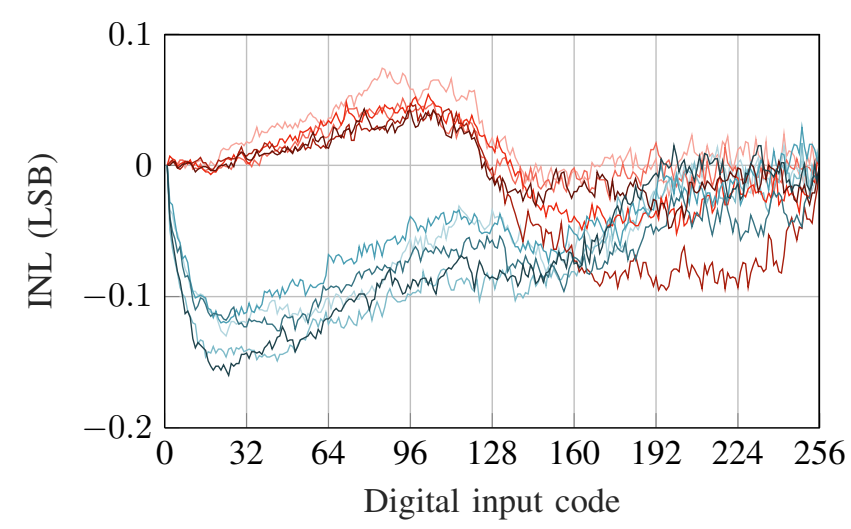

(b)

Fig. 7. Measured DNL and INL performance of one chip. Color shading distinguishes different positions in the output range as illustrated in Fig. 6.

level and stage 2 generated the ac component. The sample rate was approximately $100 \mathrm{kHz}$. The SNDR was calculated over a bandwidth of half the sample rate.

As the DAC is part of a potentiostat, the power consumption of the DAC itself cannot be measured. Post-layout simulation results estimate that the maximum power consumption is $132 \mu \mathrm{W}$ from a $3 \mathrm{~V}$ supply. The output signal frequency and the sample rate were set to $10 \mathrm{kHz}$ and $100 \mathrm{kHz}$, respectively.

A comparison of our design to selected potentiostats is shown in Table I. The output voltage range to supply voltage ratio of our DAC is superior.

TABLE I

COMPARISON OF SELECTED IMPLEMENTATIONS

\begin{tabular}{cccc}
\hline & This work & {$[24]$} & {$[25]$} \\
\hline Supply voltage (V) & 3 & 1.8 & 3 \\
Output voltage & $\mathbf{0 . 3 2}-\mathbf{2 . 6 1}{ }^{\text {a }}$ & - & $0.2-2^{\text {a }}$ \\
range (V) & $\mathbf{2 . 2 9}$ & 0.8 & 1.8 \\
Resolution (bit) & 16 & 8 & 10 \\
\hline
\end{tabular}

${ }^{\text {a }}$ Measured

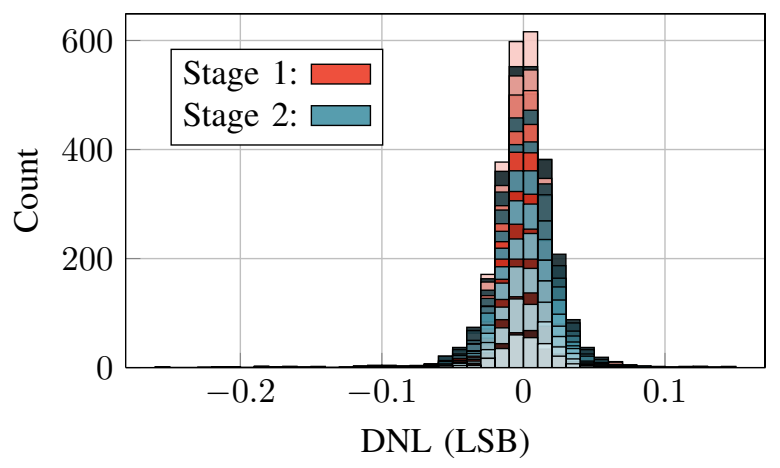

(a)

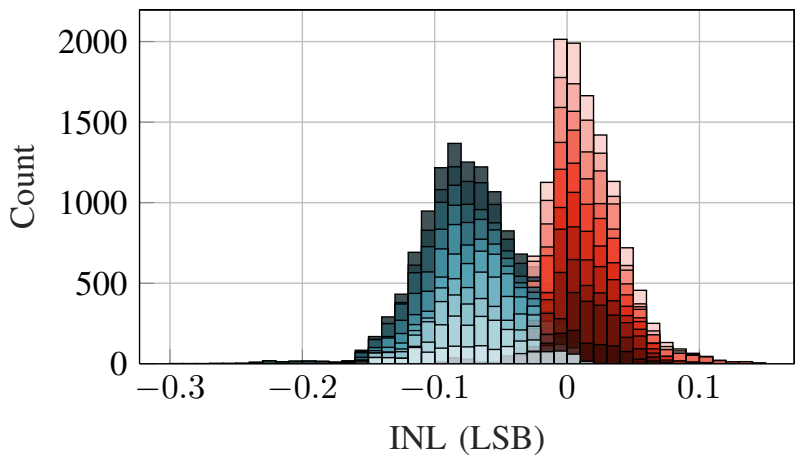

(b)

Fig. 8. Measured DNL and INL performance over 10 chips, for both Rstring stages. The histogram shows accumulated data while in different floating modes shifted over the output range, as shown in Fig. 6.

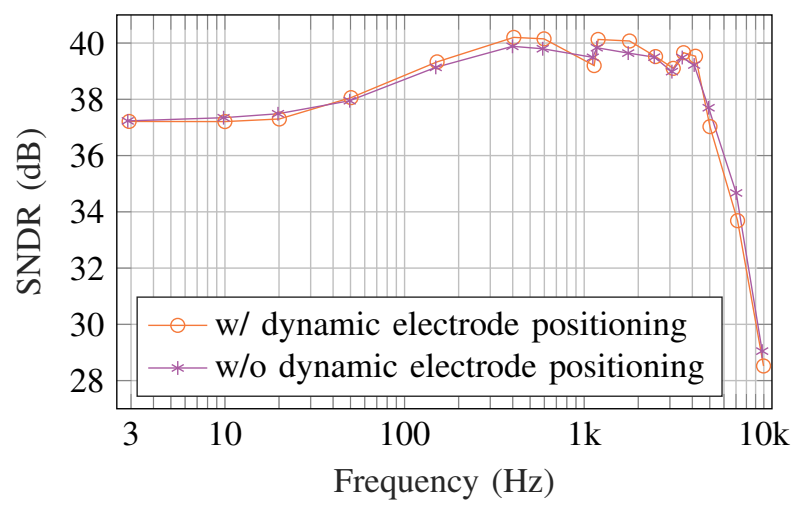

Fig. 9. Measured SNDR of the floating differential DAC. Dynamic electrode positioning does not change the performance significantly.

\section{Conclusion}

Our floating differential DAC meets the versatile requirements of an integrated potentiostat with dynamic electrode positioning. The presented DAC expands the output voltage range compared to a conventional architecture. Output signals from $0.32 \mathrm{~V}$ to $2.61 \mathrm{~V}$ with a 16 bit resolution can be generated when operated with a supply voltage of $3 \mathrm{~V}$. Measurement results show that the static and the dynamic performance of the DAC are not influenced by the output voltage range expansion. These results enable portable and low-cost electrochemical measurement solutions for POC diagnostics. 


\section{REFERENCES}

[1] Allen J. Bard, Larry R. Faulkner, Electrochemical Methods: Fundamentals and Applications. John Wiley \& Sons, INC., 2001, ISBN: 04710437929.

[2] A. A. I. of Technology GmbH. "ELSAH Research Project." (2019), [Online]. Available: http://www.elsah.researchproject.at/index.php (visited on 08/13/2021)

[3] Y. Hu, S. Sharma, J. Weatherwax, A. Cass, and P. Georgiou, "A portable multi-channel potentiostat for real-time amperometric measurement of multi-electrode sensor arrays," in 2016 IEEE International Symposium on Circuits and Systems (ISCAS), 2016, pp. 13061309, ISBN: 2379-447X

[4] L. Fujcik, R. Prokop, J. Prasek, J. Hubalek, and R. Vrba, "New CMOS potentiostat as ASIC for several electrochemical microsensors construction," Microelectronics International, vol. 27, no. 1, pp. 3-10, 2010, ISSN: 1356-5362. DOI: 10.1108/13565361011009450.

[5] C. Huang, H. Huang, and R. Yuan, "Design of a Portable Mini Potentiostat for Electrochemical Biosensors," in 2017 IEEE 2nd Advanced Information Technology, Electronic and Automation Control Conference (IAEAC), 2017, pp. 200-203.

[6] C.-Y. Huang, M.-F. Sun, and H.-Y. Lin, "Design of a Potentiostat with Standalone Signal Generator for Vanillylmandelic Acid Biosensors," in 2016 International Symposium on Computer, Consumer and Control (IS3C), IEEE, 4.07.2016 - 06.07.2016, pp. 606-609, ISBN: 978-1-5090-3071-2. DOI: 10.1109/IS3C.2016.156.

[7] M. M. Ahmadi and G. A. Jullien, "A wireless-implantable microsystem for continuous blood glucose monitoring," IEEE transactions on biomedical circuits and systems, vol. 3, no. 3, pp. 169-180, 2009, ISSN: 1932-4545. DOI: 10.1109/TBCAS.2009.2016844.

[8] M. Razzaghpour, S. Rodriguez, E. Alarcon, and A. Rusu, "A highlyaccurate low-power CMOS potentiostat for implantable biosensors," in 2011 IEEE Biomedical Circuits and Systems Conference (BioCAS), IEEE, 10.11.2011 - 12.11.2011, pp. 5-8, ISBN: 978-1-4577-1470-2. DOI: 10.1109/BioCAS.2011.6107713.

[9] D. de Venuto, M. D. Torre, C. Boero, S. Carrara, and G. de Micheli, "A novel multi-working electrode potentiostat for electrochemical detection of metabolites," in 2010 IEEE Sensors, IEEE, 1.11.2010 04.11.2010, pp. 1572-1577, ISBN: 978-1-4244-8170-5. DOI: $10.1109 /$ ICSENS.2010.5690297

[10] Byounghyun Lim, Masato Futagawa, Sou Takahashi, Fumihiro Dasai, and Kazuaki Sawada, "Integrated Square Wave Voltammetry Redox Sensor System for Electrochemical Analysis," Japanese Journal of Applied Physics, vol. 52, no. 11, 2013, ISSN: 0021-4922. DOI: 10. 7567/JJAP.52.116502. [Online]. Available: https://www.researchgate. net / publication / 270762393_Integrated_Square_Wave_Voltammetry_ Redox_Sensor_System_for_Electrochemical_Analysis.

[11] Y. Chao, H. Yue, B. L. Hassler, R. M. Worden, and A. J. Mason, "Amperometric electrochemical microsystem for a miniaturized protein biosensor array," IEEE transactions on biomedical circuits and systems, vol. 3, no. 3, pp. 160-168, 2009, ISSN: 1932-4545. DOI: 10.1109/TBCAS.2009.2015650.

[12] R. Genov, M. Stanacevic, M. Naware, G. Cauwenberghs, and N. Thakor, "16-Channel Integrated Potentiostat for Distributed Neurochemical Sensing," IEEE Transactions on Circuits and Systems I: Regular Papers, vol. 53, no. 11, pp. 2371-2376, 2006, ISSN: 10577122. DOI: $10.1109 /$ TCSI.2006.884425.

13] T. Luo, L. Li, V. Ghorband, Y. Zhan, H. Song, and J. B. Christen, "A portable impedance-based electrochemical measurement device," in 2016 IEEE International Symposium on Circuits and Systems (ISCAS), IEEE, 22.05.2016 - 25.05.2016, pp. 2891-2894, ISBN: 9781-4799-5341-7. DOI: 10.1109/ISCAS.2016.7539197.

[14] H. Jafari, L. Soleymani, and R. Genov, "16-Channel CMOS Impedance Spectroscopy DNA Analyzer With Dual-Slope Multiplying ADCs," IEEE Transactions on Biomedical Circuits and Systems, vol. 6, no. 5, pp. 468-478, 2012, ISSN: 1932-4545. DOI: 10.1109/ TBCAS.2012.2226334.

[15] H. Kim and B. Bakkaloglu, "A CMOS Analog Front-End interface IC for gas sensors," in 2010 2nd Circuits and Systems for Medical and Environmental Applications Workshop (CASME), IEEE, 13.12.2010 - 15.12.2010, pp. 1-4, ISBN: 978-1-4244-9994-6. DOI: $10.1109 /$ CASME.2010.5706689

[16] I. Kim, H. Jin, Y. Jiang, and Y. Qin, "The Optimization of Ana$\log$ Front-End for Fully Integrated Wearable Sweat Sensor," in
201941 st Annual International Conference of the IEEE Engineering in Medicine and Biology Society (EMBC), IEEE, 23.07.2019 27.07.2019, pp. 1123-1126, ISBN: 978-1-5386-1311-5. DOI: 10.1109/ EMBC.2019.8857939.

[17] Y.-T. Liu, M. Chen, Z.-C. Li, Y. Wang, and J. Chen, "A high dynamic range analog-front-end IC for electrochemical amperometric and voltammetric sensors," Microelectronics Journal, vol. 46, no. 8 pp. 716-722, 2015, ISSN: 00262692. DOI: 10.1016/j.mejo.2015.05 009.

[18] Madhi Rajabzadeh, Joachim Becker, Maurits Ortmanns, Evaluation of Single-Bit Sigma-Delta Modulator DAC for Electric Impedance Spectroscopy. Piscataway, NJ: IEEE, 2017, ISBN: 9781509058037. [Online]. Available: http : / / ieeexplore . ieee . org / servlet / opac ? punumber $=8303622$

[19] A. H. Najafabadi, A. Tamayol, N. Annabi, et al., "Biodegradable nanofibrous polymeric substrates for generating elastic and flexible electronics," Advanced materials (Deerfield Beach, Fla.), vol. 26 , no. 33, pp. 5823-5830, 2014. DOI: 10.1002/adma.201401537.

[20] R.J. Reay, S.P. Kounaves, and G.T.A Kovacs, "An integrated CMOS potentiostat for miniaturized electroanalytical instrumentation," 1994

[21] R. Turner, D. J. Harrison, and H. P. Baltes, "A CMOS potentiostat for amperometric chemical sensors," IEEE Journal of Solid-State Circuits, vol. 22, no. 3, pp. 473-478, 1987, ISSN: 0018-9200. DOI: 10.1109/JSSC.1987.1052753.

[22] M. Vergani, M. Carminati, G. Ferrari, et al., "Multichannel bipotentiostat integrated with a microfluidic platform for electrochemical realtime monitoring of cell cultures," IEEE transactions on biomedical circuits and systems, vol. 6, no. 5, pp. 498-507, 2012, ISSN: 1932 4545. DOI: $10.1109 /$ TBCAS.2012.2187783.

[23] A. Ainla, M. P. S. Mousavi, M.-N. Tsaloglou, et al., "Open-Source Potentiostat for Wireless Electrochemical Detection with Smartphones," Analytical chemistry, vol. 90, no. 10, pp. 6240-6246, 2018. DOI: 10.1021/acs.analchem.8b00850.

[24] K. Krorakai, S. Klangphukhiew, S. Kulchat, and R. Patramanon, "Smartphone-Based NFC Potentiostat for Wireless Electrochemical Sensing," Applied Sciences, vol. 11, no. 1, p. 392, 2021. DOI: 10.3390/ app11010392. [Online]. Available: https://www. mdpi .com/2076$3417 / 11 / 1 / 392$.

[25] T. K. Yew, Y. Yusoff, L. K. Sieng, H. C. Lah, H. Majid, and N. Shelida, "An electrochemical sensor ASIC for agriculture applications," in 2014 37th International Convention on Information and Communication Technology, Electronics and Microelectronics (MIPRO), IEEE, 26.05.2014 - 30.05.2014, pp. 85-90, ISBN: 978-953233-077-9. DOI: 10.1109/MIPRO.2014.6859538.

[26] Inge Siegl, Carolin Kollegger, Clemens Rabl, Christoph Steffan, Wolfgang Pribyl, "NFC Powerd Cyclic Voltammetry with Dynamic Output Voltage Range Exploitation," in 2018 Twelfth International Conference on Sensing Technology (ICST), pp. 1-3.

[27] J. Gu, H. Yao, K. Wang, B. Parviz, and B. Otis, "A 10mA on-chip electrochemical impedance spectroscopy system for wearables/implantables," in 2014 IEEE Asian Solid-State Circuits Conference (A-SSCC), IEEE, 10.11.2014 - 12.11.2014, pp. 309-312, ISBN 978-1-4799-4089-9. DOI: 10.1109/ASSCC.2014.7008922.

[28] M. Haberler, I. Siegl, C. Steffan, and M. Auer, "A Bidirectional Current-Mirror-Based Potentiostat Using a Slice-Based Class-AB Amplifier,' IEEE Solid-State Circuits Letters, vol. 3, pp. 298-301, 2020. DOI: \url\{10.1109/LSSC.2020.3013667\}. 